\title{
The Epidemiology and Phenotypes of Ocular Manifestations in Childhood and Juvenile Myasthenia Gravis: A Review
}

\author{
Jeannine M. Heckmann ${ }^{1,2 *}$, Tarin A. Europa ${ }^{1,2}$, Aayesha J. Soni ${ }^{1}$ and Melissa Nel ${ }^{2}$ \\ ${ }^{1}$ Division of Neurology, Department of Medicine, University of Cape Town, Cape Town, South Africa, ${ }^{2}$ Neurology Research \\ Group, University of Cape Town (UCT) Neuroscience Institute, University of Cape Town, Cape Town, South Africa
}

OPEN ACCESS

Edited by:

Hai-Feng Li,

Capital Medical University, China

Reviewed by:

Chongbo Zhao,

Fudan University, China

Lorenzo Maggi,

IRCCS Carlo Besta Neurological Institute Foundation, Italy

*Correspondence:

Jeannine M. Heckmann jeanine.heckmann@uct.ac.za

Specialty section:

This article was submitted to Neuromuscular Disorders and

Peripheral Neuropathies,

a section of the journal

Frontiers in Neurology

Received: 13 December 2021

Accepted: 24 January 2022

Published: 23 February 2022

Citation:

Heckmann JM, Europa TA, Soni AJ and Nel M (2022) The Epidemiology

and Phenotypes of Ocular

Manifestations in Childhood and Juvenile Myasthenia Gravis: A Review.

Front. Neurol. 13:834212.

doi: 10.3389/fneur.2022.834212
Myasthenia gravis (MG) appears to have a similar incidence among adult populations worldwide. However, epidemiological and phenotypic differences have been noted among children and juveniles with MG. We reviewed the literature on childhood- and juvenile-onset MG among different populations, with the focus on ocular involvement, antibody profiles, the genetic susceptibility to juvenile MG phenotypes, the use of immune treatments, and the reported responses of extraocular muscles to therapies. Although epidemiological studies used different methodologies, reports from Asia, compared to Europe, showed more than two-fold higher proportions of prepubertal onset (before 12 years) vs. postpubertal-onset juveniles with MG. Compared to European children, ocular MG was 4-fold more frequent among Asian children, and 2-3-fold more frequent among children with African ancestry both in prepubertal and postpubertal ages at onset. These results suggest genetic influences. In Asia, HLA-B*46 and $D R B 1^{*} 09$ appeared overrepresented in children with ocular MG. In Europe, children with $M G$ had a significantly higher rate of transforming from ocular to generalized disease and with an overrepresentation of HLADRB1*04. Although treatment regimens vary widely and the responses to immune therapies of the ocular muscles involved in MG were generally poorly described, there were indications that earlier use of steroid therapy may have better outcomes. Reports of treatment-resistant ophthalmoplegia may be more frequent in African and Asian juvenile MG cohorts compared to Europeans. Genetic and muscle gene expression studies point to dysregulated muscle atrophy signaling and mitochondrial metabolism pathways as pathogenetic mechanisms underpinning treatment-resistant ophthalmoplegia in susceptible individuals. In conclusion, phenotypic differences in juveniles with ocular manifestations of $M G$ were evident in different populations suggesting pathogenetic influences. Treatment responses in MG-associated ocular disease should attract more careful descriptive reports. In MG, extraocular muscles may be vulnerable to critical periods of poor force generation and certain individuals may be particularly susceptible to developing treatment-resistant ophthalmoplegia. The development of prognostic biomarkers to identify these susceptible individuals is an unmet need.

Keywords: treatment refractory ophthalmoplegia, ocular myasthenia gravis (OMG), childhood myasthenia gravis, juvenile myasthenia gravis, genetic susceptibility, Asian ancestry, myasthenia (myasthenia gravis-MG), African ancestry 


\section{BACKGROUND}

Myasthenia gravis (MG) represents a heterogeneous group of autoantibody-mediated diseases targeting the neuromuscular junction. Extraocular muscles (EOMs) are highly susceptible to manifesting myasthenic weakness and are frequently involved early in the MG disease course prior to developing generalized myasthenia $(1,2)$. Younger children appear to have a higher prevalence of developing ocular MG (myasthenia remains confined to the EOMs for an extended period) among Asian cohorts, but the outcomes of EOMs to MG therapies are generally not adequately described. This study aimed to review the epidemiological literature of childhood and juvenile MG and determine the severity of ocular phenotypes and treatment responses, as well as current postulates related to the pathogenetic mechanisms underlying the ocular phenotypes with the focus on, but not confined to, the past decade.

\section{METHODS}

\section{Search Strategy and Selection Criteria Epidemiological Data}

We searched the PubMed database for reports published in English between January 1, 2010 and October 31, 2021 with the MeSH terms "juvenile" or "childhood" in combination with "myasthenia gravis," "ocular myasthenia gravis," and "antibody." We also selected references from manual searches of reference lists of articles and reviews. Some of these references were published before 2010, but after 1991. We included publications that had clearly stated diagnostic criteria and in which epidemiological data could be extracted such as age at onset and phenotypic characteristics such as acetylcholine receptor (AChR)-antibody status, ocular involvement, secondary generalization, frequency of autoimmune diseases and thymoma, sex differences, and outcomes of ocular myasthenia. If there were 2 publications from the same group, then we included only the most recent article unless unique data was mentioned in the first report. For juvenile MG, we included reports specifying age at onset of MG symptoms between 1 and 20 years, despite the most frequent age cutoff for juvenile onset MG being < 18 years (3).

\section{Genetic Data}

Search terms included; "gene" or "HLA" and "ocular myasthenia," "juvenile myasthenia gravis," "childhood myasthenia gravis," and "extraocular muscles." We also searched using geographical terms "Asia," "China," "Africa," and "myasthenia gravis." Original research articles written in English and published between 1996 and October 2021, which compared MG/MG subgroup vs. age and race matched healthy controls, were selected for review particularly if there was a special reference to EOM involvement at presentation, treatment approaches, and descriptive outcomes to treatment. When appropriate data were extracted for positive individual human leukocyte antigen (HLA) associations (excluding haplotypes) with MG (by subgroup if specified).

\section{Data Extraction and Organization}

Although the use of critical appraisal tools to judge the scientific merit of studies for inclusion in a review is encouraged, the scarcity of studies including adequate descriptions of ocular manifestations made the use of such tools difficult to implement. A further limitation was the heterogeneity of age cutoffs for juveniles, childhood, prepubertal and postpubertal cases with MG; while most reports define the age of 12 years as the threshold of puberty and $<18$ years as juvenile onset, there were different cutoffs to differentiate postpubertal MG from early-onset adult MG, and childhood MG from prepubertal MG. These were indicated as per author(s) and grouped together for comparative purposes (Table 1).

\section{RESULTS}

\section{Population Differences in MG by age at Symptom Onset}

Although there is recognition worldwide of an increasing predominance of MG among the elderly, including in Asia and Africa (4, 21-25), incidence rates among younger people manifesting with MG appear to differ between Asia and Europe. Population data including children are sparse and methodologies vary widely, but there appear to be four-fold higher incidence rates of MG among younger children from Asia compared to Europe and North America (12, 19, 26). A multiracial pediatric cohort from the United Kingdom (UK) in which data were accrued over 10 years showed similar findings with higher proportions of Afro-Caribbean, Asian, and Arabic children with MG compared to Caucasian children living in the UK (20).

Reports from China regarding the proportions of juveniles with MG, vary substantially and ranged between $27 \%(302 / 1,108)$ in northern China, and 45\% $(964 / 2,154)$ in southern China (27) (Table 1). Nevertheless, at least half of the children manifested with MG before the age of 10 , and the incidence peaked in those presenting with symptoms before the age of 5 years (6, 8) (Table 1). A nationwide MG prevalence questionnaire from Japan showed that children developing MG before the age of 10 years accounted for $9 \%$ of the overall proportion of MG cases $(n=3,061)$ (4), which is much lower compared to China, but remains substantially higher than the $2 \%$ prevalence in Italy (13). Therefore, despite the possible impact of differences in study methodology on the epidemiological results, the incidence of MG in both the prepubertal and postpubertal juveniles, compared to adult-onset disease, was lower in juveniles with European genetic ancestry compared to those with Asian and African genetic ancestry.

\section{Population and Phenotype Differences Among Categories of Juveniles With MG Prepubertal vs. Postpubertal Onset}

There is accumulating evidence that MG presenting in the prepubertal phase in contrast to postpubertal onset differs by genetic ancestry. Studies from Asia showed the proportions of children developing myasthenia before puberty $(\geq 74 \%)$ were more than twice as high compared to postpubertal children, and 
TABLE 1 | Characteristics of juvenile myasthenia gravis (MG) and subgroups (pre-pubertal vs. post-pubertal) by race and/or geographical area.

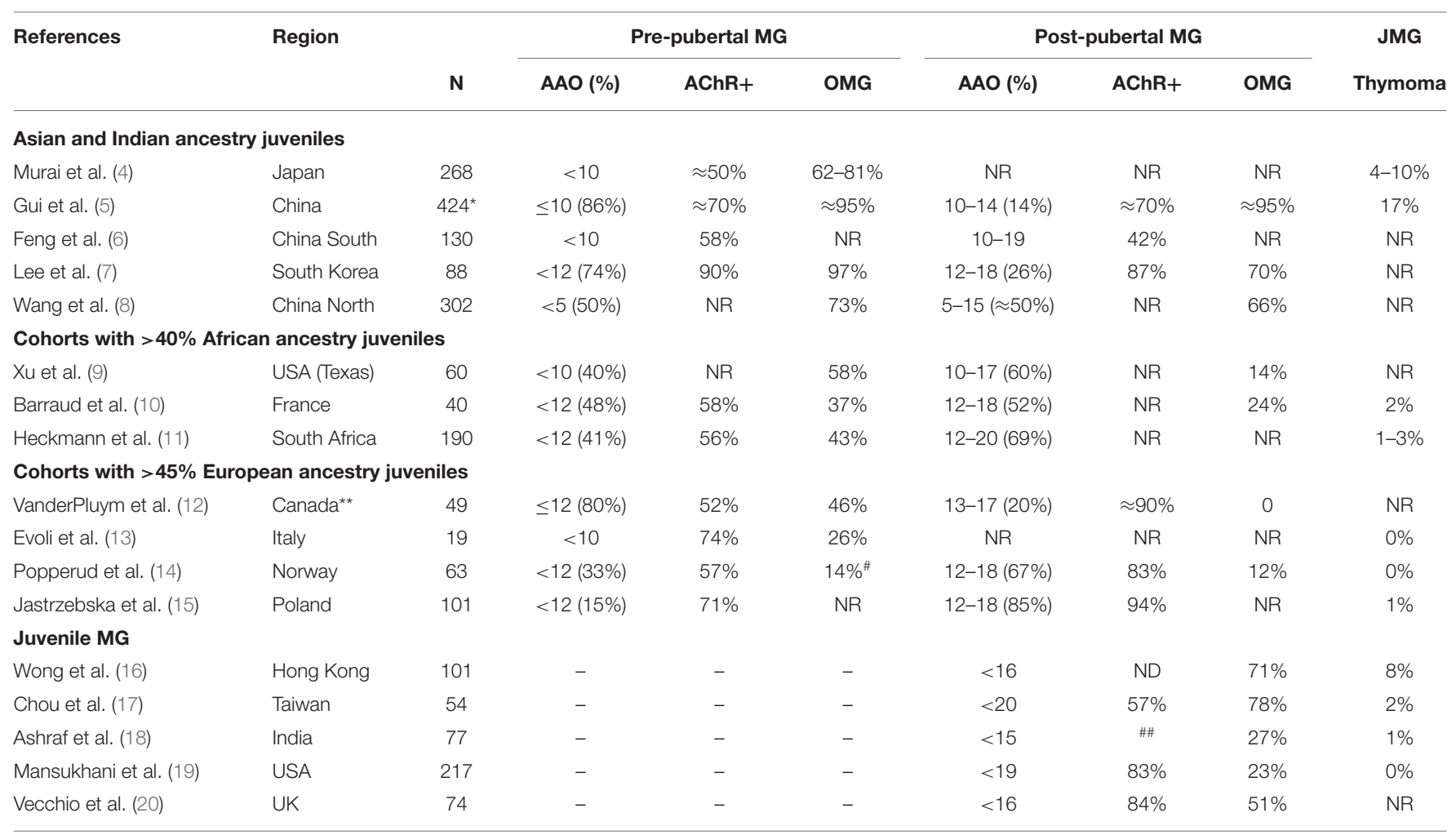

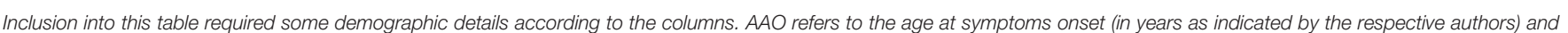

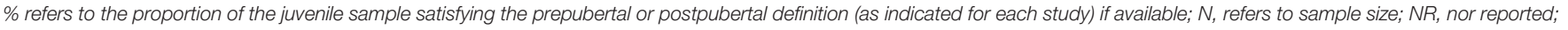

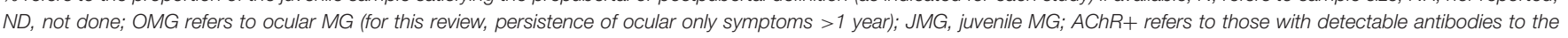
acetylcholine receptor.

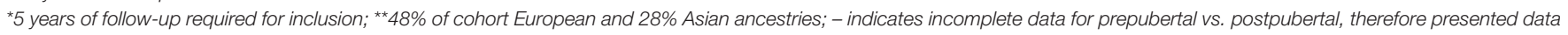
as juvenile MG. " used the follow-up data. \#\# AChR+ data only available for 18\% (11/14 AChR+).

contrasts with a more even distribution ( $\sim 40$ to $48 \%)$ amongst cohorts with African children, and $<33 \%$ in cohorts comprising European children (Table 1). A large cohort from China showed that half of the juveniles developing MG before age 15 were younger than 5 years (8).

In Asia, there was a definite tendency toward more ocular MG amongst the very young, prepubertal children compared to older aged children with MG, but this was not evident in the Norwegian children (Table 1). A multiracial juvenile MG cohort from Canada, in which only $48 \%$ had European ancestry, also showed a much higher proportion of prepubertal onset MG, and most of the very young onset ocular MG cases (aged $\leq 6$ years) had Asian ancestry (12).

Interestingly, two multiracial cohorts from France $(48 \%$ of 40 had African ancestry) (10) and the UK (54\% of 74 did not have European ancestry) (20) showed similar results in which prepubertal ocular MG were more likely in the African children despite equal proportions of children with pre- and postpubertal MG. A feature of MG among north European children (Norway and Italy) was that ocular only presentations of MG occurred in less than a third, with most children (>75\%) developing generalized disease (with/or without respiratory involvement) within 2 years, and between 15 and $26 \%$ remained with ocular MG $(13,14)$. Similar observations were noted in Canada where white children were more likely to develop generalized MG, and Asian children remained with ocular disease (12). Furthermore, the conversion of ocular MG cases to generalized disease was reported in only 5 to $20 \%$ of Chinese and Thai children $(5,16,28,29)$ and among $25 \%$ of the French cohort in which almost half the children had African genetic ancestry (10).

Sex differences and severity of MG were not consistently different in postpubertal cohorts from different populations; a European cohort showed more girls in the postpubertal group with less severe MG disease (14); two Asian cohorts showed similar proportions of girls and boys, but inconsistent severity of MG grades by sex were reported $(7,28)$. An older study from the USA, which specifically assessed MG outcomes by race in a clinical setting where the same treatment approaches were used for all children, reported infrequent clinical remissions in prepubertal black patients compared to white patients, although overall disease severity was similar irrespective of race (30). It is important to highlight that MG crises can occur in children and require appropriate immune therapies $(3,12,26)$.

In summary, pre- and postpubertal MG cases were more likely to remain confined to the ocular muscles in Asian children compared to those in Europe. 


\section{Antibody Profile}

The AChR-Ab positive MG frequencies by RIA appeared to be similar in all children and in almost all studies ranged between 50 and 95\%, irrespective of whether the MG onset was prepubertal or postpubertal (Table 1). A study from China found similar proportions of AChR-Abs by RIA and cell-based assay (CBA) in juveniles ( $<19$ years of age) compared to adult-onset MG cases, although $18 \%$ of the juveniles (compared to $10 \%$ of adults) were only positive by CBA (31). The age-adjusted incidence rates of AChR-Ab positive MG among juveniles from South Africa (24) appeared to be higher than in Caucasian cohorts from the UK, USA, Norway, and Canada $(\approx 3$ per million vs. $<1.5$ per million, respectively) $(12,19,32,33)$.

Data on the prevalence of muscle-specific kinase (MuSK)-Abs are sparse. Only rare cases of MuSK-Ab positive MG have been reported in juveniles from China [0/118 (31) or < 3\% (6)], Japan (1.4\%) (4), North America (34), and northern Europe (35) and possibly more than expected in the two cohorts with African ancestry children $(10,14,15,17,19,20)$.

Overall, most populations reported that younger children were more likely to have AChR-Ab negative MG and ocular disease, both of which conferred a higher likelihood of obtaining remission status $(17,20,28)$. However, in the situation where the child does not respond to treatment, despite symptom onset after infancy, the question of possible congenital myasthenia may arise. Clinical features supportive of autoimmune MG include: subacute progressive onset; marked asymmetry of ptosis; substantial fluctuations of ophthalmoplegia (36).

\section{Thymoma Incidence}

Thymoma occurs rarely in juveniles with MG (34). Data from Asia varied between 0 (0/118) (31) and 17\% (6/34) (Table 1).

\section{Autoimmune Disease}

Concomitant autoimmune disease, mainly thyroid disease, was reported in $4-19 \%$ of children with MG from China, Thailand, Hong Kong, and racially diverse cohorts from Canada and the UK $(4,5,12,16,20,27-29), 27 \%$ from Taiwan (included MG onset before age 20) (17) and $\approx 7 \%$ in juvenile cohorts with substantial African ancestry MG cases $(10,11)$. In contrast, $\approx 30 \%$ of pre- and postpubertal Norwegian children had other autoimmune diseases in addition to MG (14).

\section{Epidemiology of Ocular MG Among Juveniles}

The higher frequencies of ocular MG among younger children from Asia differed substantially from Europe $(4,22,28)$ (Table 1). Within the prepubertal onset range, the very young children presenting with symptoms before the age of 4 , showed the highest proportions of ocular MG compared to older children from China and Japan $(4,23,28)$. African, Afro-Caribbean, and African-American prepubertal onset children also showed higher proportions of ocular MG compared to postpubertal juveniles $(9,11,12,20)$.

\section{Severity of Extraocular Muscle Involvement at the Presentation of MG in Juveniles vs. Adults}

There was a paucity of descriptive data of EOM involvement in MG. An audit of the examination findings in adults presenting with MG to a Scottish ophthalmological service, reported bilateral weakness of multiple EOMs in more than half the patients, irrespective of age, with $6 \%$ having bilateral ophthalmopareses (or duction failure) (37). A review from Thailand, but in juveniles ( $<15$ years) presenting with ocular MG, also found limitations of EOM movement in more than $50 \%$ (of 62), and most had complete duction failure (29). Juvenile MG cases seen at the Mayo clinic (most were Caucasian children) found limitations of EOM movement in 30\%, although there may be a bias to more severe cases in this cohort as most patients were not residents of the county (19).

Observational descriptive EOM data from a largely adult MG clinic, prior to any therapy and in which $\approx 15 \%$ had only ocular manifestations of $\mathrm{MG}$, showed that $\approx 12 \%$ of $\mathrm{MG}$ cases had fatigable ptosis/diplopia compared to $\approx 87 \%$ with persistent ophthalmoparesis (or weakness) with or without ptosis in at least one EOM (38). Of those with ophthalmoparesis, $>60 \%$ had weakness of $\geq 6$ EOMs. There was a trend toward more severe weakness in those with generalized MG compared to ocular only MG (severity is defined by the number of EOMs with $\geq 50 \%$ weakness (i.e., can only move half of the EOM's full trajectory) (38). It is worth mentioning that even mild weakness of one EOM may cause diplopia, and those patients with complete ophthalmoplegia may not experience diplopia, although minor malignment of the visual axes may result in diplopia (39).

Taken together, a substantial proportion of patients with MG may develop persistent weakness of their EOMs (ophthalmoparesis or ophthalmoplegia), and this may occur more frequently in juveniles. However, the absence of a standardized approach to reporting does not allow for firm conclusions (see below).

\section{Treatment Outcomes of Extraocular Muscles in MG}

The quantitative and descriptive data with respect to EOM outcomes to therapy in juveniles with MG, were sparse, highlighting a research gap (Table 2). A large cohort of 306 juveniles with ocular MG from southern China, of whom most were treated with immune therapy in addition to pyridostigmine, only $50 \%$ achieved minimal manifestations (43) or better after at least 12 months of follow-up (28). Better outcomes were related to earlier use of "standard treatment" (within 2 years of symptom onset), which included the use of prednisone 0.25 $\mathrm{mg} / \mathrm{kg} /$ day if symptoms did not resolve with pyridostigmine alone, followed by a slow taper and steroid cessation after 6 months of clinical remission (28). Another large study from China, in which $95 \%$ of juveniles had ocular involvement, only $17 \%$ "improved" while the remainder were either unchanged or worse, despite immune treatments (advising prednisone 0.75 
TABLE 2 | Outcomes of extraocular muscles in juveniles with MG by region.

\begin{tabular}{|c|c|c|c|c|c|c|}
\hline & Region & AAO, years & $\mathbf{N}$ & Follow-up, years (mean) & $\begin{array}{l}\text { Ocular outcomes: good vs. } \\
\text { treatment resistance as } \%\end{array}$ & $\begin{array}{l}\text { OMG patients on } \\
\text { immune treatment }\end{array}$ \\
\hline Kim et al. (40) & S/Korea & $<15$ & 24 & 3.1 & NR; $10 \%$ TRO & $75 \%$ \\
\hline Lee et al. (7) & S/Korea & $<18$ & 88 & $>2.6$ & $65 \%$ vs. 0 & $>55 \%$ \\
\hline Kraithat et al. (41) & Thailand & $<15$ & 14 & 6.3 & $93 \%$ vs. $7 \%$ & $79 \%$ \\
\hline Vanikieti et al. (29) & Thailand & $<15$ & 62 & $>4$ & $\mathrm{NR} ; 8 \% \mathrm{TRO}$ & $52 \%$ \\
\hline Huang et al. (28) & China & $<18$ & 306 & $>1$ & NR; $50 \%$ in remission & $93 \%$ \\
\hline Gui et al. (5) & China & $<14$ & 424 & $>5$ & NR; most unchanged/worse & $100 \%$ \\
\hline Ortiz and Borchert (42) & US & $<12$ & 21 & 6.5 & NR; OMG resolved in 19\% & $29 \%$ \\
\hline Xu et al. (9) & US & $<18$ & 22 & NR & NR; 0 TRO & "Almost all" \\
\hline
\end{tabular}

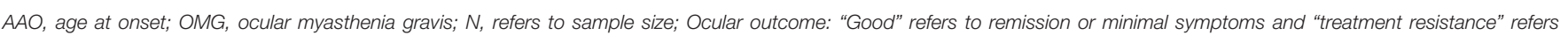
unchanged or worse; TRO refers to treatment-resistant ophthalmoplegia. S/Korea, South Korea; NR refers to not reported.

$\mathrm{mg} / \mathrm{kg} /$ day with poor responses to pyridostigmine), and even thymectomies (5).

The retrospective results of hospital-based pediatric clinics in South Africa showed, after a median follow-up of 5 years, $31 \%$ of prepubertal children $(n=31)$ remained with partial or complete treatment-resistant ophthalmoplegia, and $12 \%$ in the postpubertal group $(n=20)(11)$. Although immune treatments were used in this case series, the treatment protocols varied from site to site. In contrast, the pediatric group from North America ( $n=22 ; 40 \%$ of children with African ancestry) in which $>80 \%$ were treated within a median of 5 months from symptom onset, and using doses of prednisone $2.5 \mathrm{mg} / \mathrm{kg} /$ day for $4-6$ weeks before a reduction to alternate day dosing, resulted in all the patients reaching minimal manifestation status or better (9).

In a cohort of predominantly adult MG patients, longitudinal observational data to assess the duration of immune treatment required before the resolution of MG-induced EOM paresis showed that starting immune therapy earlier $(<12$ months of symptom onset) and using higher doses of prednisone in the first 3 months ( 0.45 vs. $0.29 \mathrm{mg} / \mathrm{kg}$ ) associated with significantly better outcomes; patients whose ophthalmoplegia resolved within 3 months of starting therapy had received the higher dose compared to those who only showed resolution of ophthalmoplegia between 4 and 12 months (38). Although there were only nine of 76 patients with MG manifesting with MG before the age of 20 in this cohort, the younger people were less likely to show resolution at 12 months compared to the older people (statistical analyses were not performed due to sample size). Of those with EOM weakness at baseline, $24 \%$ remained with complete ophthalmoplegia (all the 12 EOMs with persistent paresis) at 12 months despite moderate doses of prednisone $\approx 0.35 \mathrm{mg} / \mathrm{kg}$ daily with/without steroid-sparing agents (38). These results support the treatment recommendations from Kupersmith and Ying to use earlier and higher doses of prednisone, up to $60 \mathrm{mg}$ daily, for short periods in treating the EOM manifestations of MG (44).

An international working group advising on therapies for juvenile MG recommended starting cholinesterase inhibitors at 0.5 to $1 \mathrm{mg} / \mathrm{kg}$ every 4 to $6 \mathrm{~h}$ and increasing the dose to $7 \mathrm{mg} / \mathrm{kg} /$ day in divided doses for symptom control (3).
In our experience, cholinesterase inhibitors may produce some symptomatic relief to the ocular manifestations of MG, especially ptosis, but rarely result in resolution of symptoms; however, others have noted that $>50 \%$ of patients improve symptomatically on cholinesterase treatment (10). Oral steroids, between 0.5 and $1 \mathrm{mg} / \mathrm{kg}$ daily (or $1.5 \mathrm{mg} / \mathrm{kg}$ alternate days), are advised in increasing doses in juveniles not responding to cholinesterase inhibitors, with lower doses advised in children with only ocular manifestations (3). Several groups recommend adding steroid-sparing agents to prednisone in children in the setting of poor treatment responses to steroids $(3,5,11$, 12, 28). Steroid-sparing agents which are used in juveniles include azathioprine, mycophenolate mofetil, and rituximab (3). Although methotrexate is increasingly accepted as a cost-effective adjunct to the MG therapeutic armamentarium in adults (45) based on decades of experience in the juvenile arthritides among others, we also use methotrexate in children $\left(10-15 \mathrm{mg} / \mathrm{m}^{2} /\right.$ week plus folic acid $>24 \mathrm{~h}$ after methotrexate (folate dose $\approx 1 / 3$ of methotrexate) (46).

\section{Differential Diagnosis for Treatment-Resistant Seronegative Ocular Myasthenia}

Treatment-refractory ophthalmoparesis/plegia among particularly the prepubertal group of juveniles with AChRAb negative $\mathrm{MG}$ or MuSK-Ab negative $\mathrm{MG}$, may raise the possibility of a congenital myasthenic syndrome (CMS). CMS usually manifests with features of fatigable ocular or generalized muscle weakness at birth or within the first year of life, and often with a family history of a similar phenotype (47). However, pathogenic variations in several CMS genes may manifest in childhood (CHNRE; COLQ; DOK7; GFPT1; RAPSN), adolescence (DPAGT1), or even in adulthood (CHRNA1; CHRNE; DOK7; GFPT1; RAPSN) (47). Most of these CMS are accompanied by additional features such as dysmorphism (CHRNA1), or limb-girdle pattern of weakness (GFPT1; GMPPB; DGPAGT1) without EOM weakness or ptosis. Pathogenic gene variants in a few CMS genes may rarely cause diagnostic confusion with "treatment resistant ocular \pm generalized myasthenia"; pathogenic variants in CHRNE1 have been reported to present after infancy with mild ptosis or 
ophthalmoplegia and respond to cholinesterase inhibitors; DOK7 pathogenic variants may present with limb-girdle weakness and ptosis; occasional pathogenic variants in RAPSN may cause fluctuating ptosis with/without generalized fatigability $(36,47)$. Although pathogenic variants in COLQ usually cause severe early onset axial weakness with sparing of EOMs, some cases may have later onset, milder disease with variable occurrence of ophthalmoplegia and ptosis; these patients do not respond to cholinesterase inhibitors (36).

\section{Treatment-Resistant Ophthalmoplegia and Definitions}

Myasthenic involvement of the EOMs, similar to non-ocular muscles, is expected to respond to immunosuppressive therapies (38). However, in 2007, we first highlighted the occurrence of chronic treatment-resistant ophthalmoparesis (or ophthalmoplegia) in a subset of patients with MG from South Africa, whereas their non-ocular muscles responded to immune therapies. Treatment-resistant ophthalmoplegia occurred more frequently in those with younger onset ( $<20$ years), AChR-Ab positive MG, and in individuals with African genetic ancestry (48). Subsequently, cross-sectional data from different pediatric centers across South Africa showed that up to 30\% of the children attending hospital-based clinics remained with degrees of ophthalmoplegia after several years of immune therapies, irrespective of whether they had ocular-only or generalized MG (11).

Although complete ophthalmoplegia (also referred to as "eyeball fixation") (6) is mentioned in juvenile cohorts from Asia, and elsewhere, it is frequently not quantified. Nevertheless, a Korean cohort of childhood-onset ocular MG (onset before 15 years and follow-up $>6$ months) reported that only $29 \%$ (of 24 cases) improved in response to treatment with pyridostigmine and prednisone and $10 \%$ of patients remained with total ophthalmoplegia; only $50 \%$ were treated with prednisone and pyridostigmine (40) (Table 2). Treatmentresistant ophthalmoplegia was also reported in cohorts from Italy (3 of 19, 15\%) and Canada (1 of 25, 4\%) comprising either childhood-onset generalized or ocular MG and was frequently treated with immunosuppressive therapies and thymectomies $(13,49)$. Children with ocular MG from the USA ( $n=21$; followed for 2 years) showed "limitation of ductions" in $81 \%$ and complete resolution of myasthenic signs occurred in only $19 \%$, although only a third had received steroids (42). Treatment resistance requiring oculoplastic surgery was reported in $6 \%$ of mainly Caucasian juveniles in another US cohort (19).

Taken together, younger African and Asian children with myasthenic involvement of EOMs appear to be at greater risk of developing treatment-resistant ophthalmoplegia (11, 29, 40). It is important to note that adult-onset MG cases, irrespective of ocular only $\mathrm{MG}$ or generalized $\mathrm{MG}$, may develop treatment-resistant ophthalmoplegia including those with $\mathrm{MuSK}-\mathrm{Ab}$ positive $\mathrm{MG}$, triple seronegative $\mathrm{MG}$, and older men with AChR-Ab positive MG $(38,50-52)$.

Presently, there is no definition for treatment-resistant or refractory ophthalmoplegia in MG. Definitions related to refractory generalized $\mathrm{MG}$ do not apply as patients with ophthalmoplegia ( \pm ptosis) may experience substantial visual disability while the remaining non-ocular muscles may not be severely weak. In addition, refractoriness in generalized disease often requires documentation of treatment non-responsiveness and failure to prevent severe generalized MG weakness or crisis after trying several immune therapies for 12 to 24 months $(53,54)$, whereas observations suggest EOMs are vulnerable to shorter periods of inactivity due to functional denervation. Therefore, the definition of treatment-resistant ophthalmoplegia cannot be conservative as waiting for long periods in this setting may be counterproductive and contribute to muscle atrophy (Figure 1). Longitudinal observations of new patients with MG with persistent ophthalmoparesis/plegia and the timing of their resolution (or not) to immune therapy suggest that a signal for treatment non-responsiveness in most cases is evident around 6-7 months (38). However, another scenario occurs in which patients with MG may only manifest treatmentresistant ophthalmoplegia later, even after initially showing treatment responsiveness of their EOMs; in these cases, usually in the context of generalized disease, we noted that a critical event (infection; abrupt non-compliance) resulted in a relapse of MG and ophthalmoplegia with ongoing persistent nonresponsiveness of the EOMs while the non-ocular muscles responded to the re-introduction/adjustments of MG therapies. We postulate that these events may have triggered critical biological pathways (see below) (39).

The clinical examination in patients with MG with chronic treatment-resistant ophthalmoplegia may also vary; some patients show an initial brief quiver movement as the saccadic movement is initiated before the eye stops short of its reduced trajectory, or brief lid twitches with attempted upgaze after a period of downgaze may be observed. However, after years of treatment-resistant ophthalmoplegia, the EOMs of some patients with MG show very limited and slow movements, and in some, there is no observable movement at all. When there is complete ophthalmoplegia, forced duction testing by an ophthalmologist may distinguish whether an apparently "fixed" eyeball can move through its trajectory; this can distinguish between severe eye muscle paralysis, where there is no mechanical restriction to forced EOM duction, and a restrictive force which prevents ocular movement (infiltration or fibrosis). In the setting of concomitant thyroid eye disease, the EOMs would show limited mechanical movement (39).

\section{Genetic Differences of Juvenile MG by Race/Population \\ Human Leukocyte Antigen Genes}

The HLA region on chromosome 6 was the first genetic region, encompassing various class I (HLA-A, HLA-B) and class II genes (HLA-DR, HLA-DQ), shown to associate with MG (55). These HLA genes encode molecules that present antigens to $\mathrm{CD} 4+\mathrm{T}$ helper cells which are necessary to mount an adaptive immune response specific to foreign pathogens [reviewed in $\mathrm{Nel}$ et al. (56)]. Although many HLA association studies have been performed in adults with MG, those in juveniles and children are sparse, but may suggest that juvenile and/or ocular MG may have 


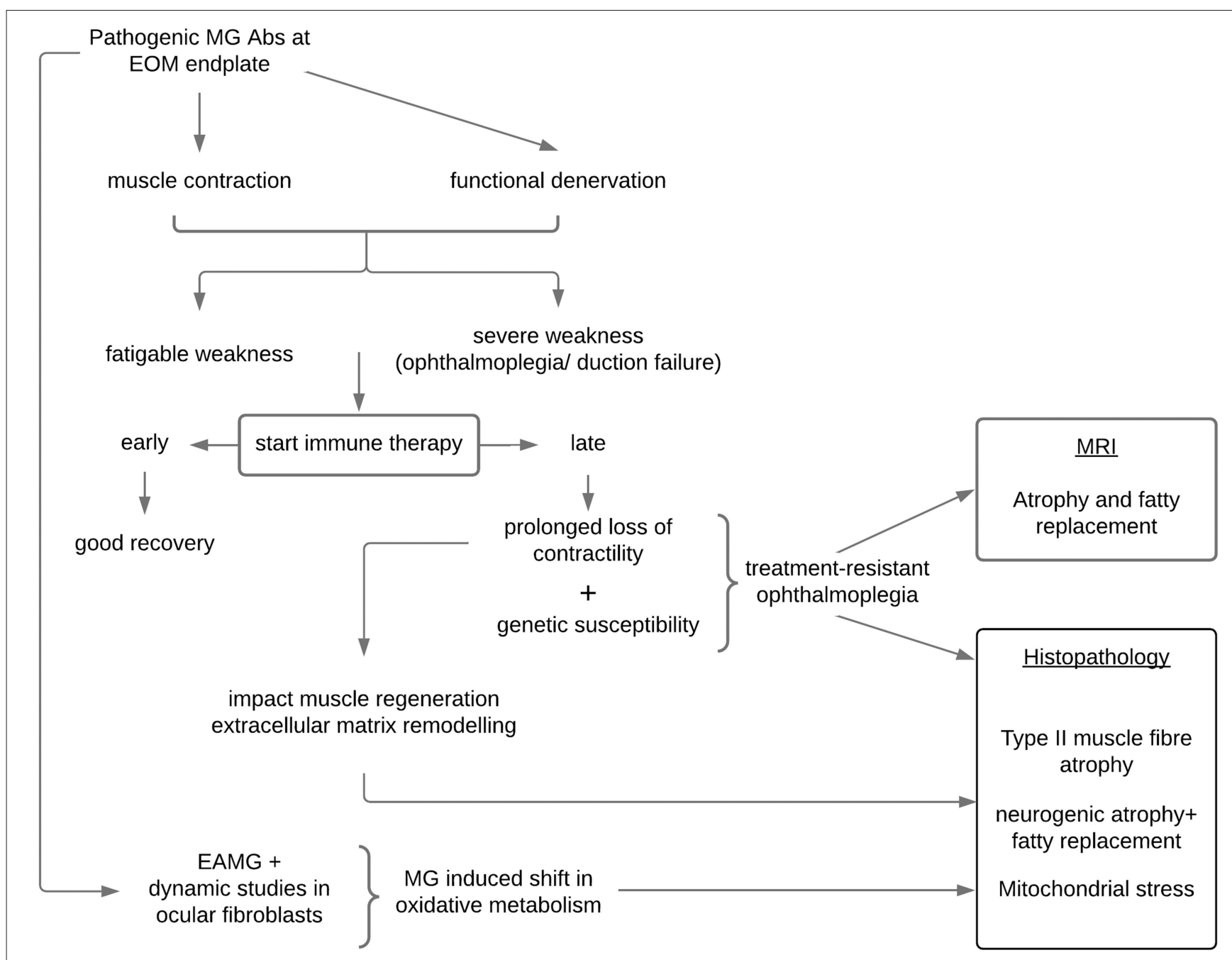

FIGURE 1 | Proposed mechanisms in the development of treatment-resistant ophthalmoplegia in susceptible patients with myasthenia gravis. EOM, extraocular muscles; MRI, magnetic resonance imaging of the orbit; EAMG, experimental autoimmune myasthenia gravis; Abs, antibodies.

a distinct immunological basis in certain populations (Table 3). For example, children from Norway showed an association with $D R B 1^{*} 04$ (61) whereas those from Asia were associated with $D R B 1^{*} 09$. Childhood-onset ocular MG in Japanese and Chinese children, who were predominantly AChR-Ab negative, have shown reasonably consistent $H L A-B^{*} 4601$; $D R B 1^{*} 0901$ associations. Nel et al., found a higher frequency of functional variants in the HLA-DRB1 region in a selected sample of African juveniles with treatment-resistant ophthalmoplegia (see below) compared to MG cases who responded to therapy (65), as well as the closely linked $H L A-D P B 1$ region (2). Preliminary results suggest that "low expression" HLA-DPB1*105:01 genotypes, which were also more common in African controls compared to European controls, associated with African juveniles with treatment-resistant ophthalmoplegia (2).

\section{Pathogenic Mechanisms of Treatment-Resistant Ophthalmoplegia in MG}

Our current hypothesis is that in a genetically susceptible individual, treatment-resistant ophthalmoplegia is likely the result of a complex network of dysregulated genes "activated" within the context of MG (39). Against this backdrop and together with a critical period of loss of contractility in the EOMs, muscle atrophy-pathways and mitochondrial metabolic pathways are not able to maintain normal homeostasis, and the paralysis of the EOMs may enter an irreversible phase of mitochondrial stress, EOM atrophy, and fat replacement (66, 67). Importantly, these histopathological changes may not be peculiar to MG, but rather to EOMs (more than other skeletal limb muscles) being particularly vulnerable to atrophy when contractility is compromised for a critical period irrespective of the cause (67). Similar to the EOM histopathological findings, imaging of the EOMs in patients with MG with chronic refractory ocular symptoms, found evidence of muscle atrophy and fatty replacement (52). Interestingly, fatty replacement with larger muscle volume was evident in the EOMs of a pilot case series (feasibility study) earlier in their disease course (68), whereas those with a longer disease duration showed muscle atrophy (69).

Gene expression studies in the EOMs of experimentally induced MG in rodents have also pointed to altered oxidative 
TABLE 3 | Human leukocyte antigen (HLA) associations in juvenile myasthenia gravis by racial ancestry or geographical area.

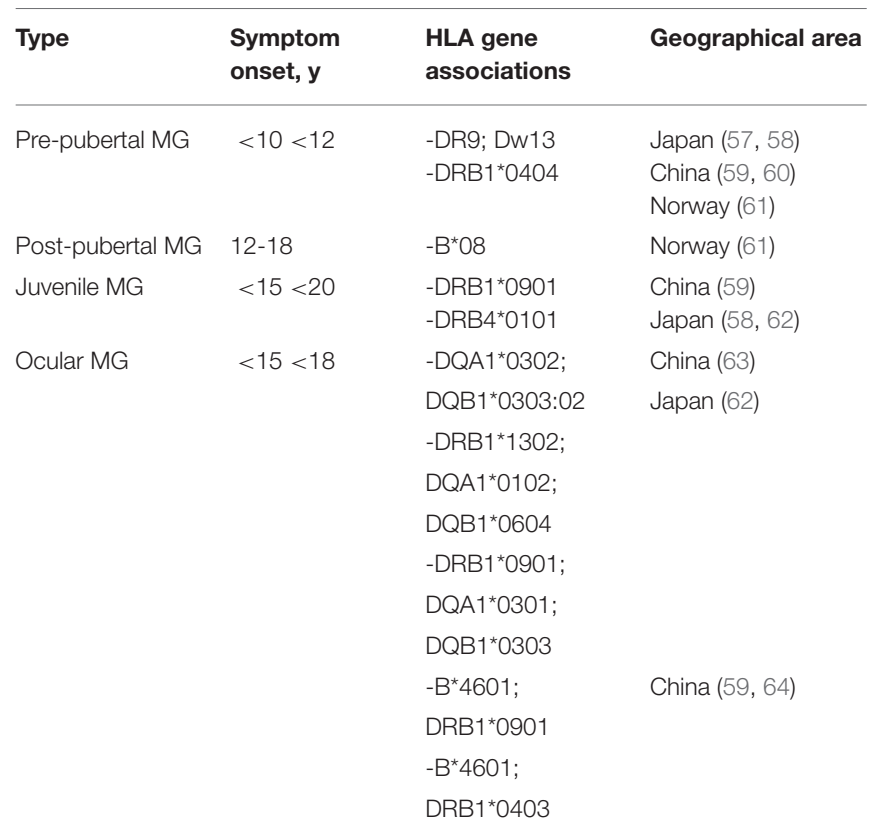

MG to myasthenia gravis. Ocular MG when MG has been confined to ocular muscles for $>2$ years. $Y$, years. Both serological and molecular HLA typing methods were considered. HLA alleles derived from molecular typing are denoted with an asterix $\left({ }^{*}\right)$ e.g., DRB1 ${ }^{\star} 0901$ is the gene for the serotype DR9. For more detail on the curation of HLA studies see Nel and Heckmann (56).

metabolism (70) which may in turn impact on EOMs maintaining high firing rates and generating contractile force (Figure 1). Poor muscle force generation affects mitochondrial biogenesis and triggers muscle atrophy signaling pathways (71, 72) all of which have been shown to be relevant in MG invitro modeling (73). The patient developing treatment-resistant ophthalmoplegia may be genetically susceptible to the induction of these "dysregulated" pathways only when they develop MG and possibly enter an irreversible stage when not treated early enough.

Although genetic studies have been limited due to the rarity of these patients, candidate gene approaches in juvenile AChR$\mathrm{Ab}$ positive generalized patients with MG with the treatmentresistant ophthalmoplegic phenotype showed associations with regulatory variants in both the DAF $(-198 C>G)$ and TGFB1 ($387 C>T)$ genes $(74,75)$. However, these genetic associations did not account for many of the cases.

An unbiased genome-wide analysis in a highly selected enriched group of juveniles with treatment-resistant ophthalmoplegic MG compared to a matched control group of young myasthenic responders (extreme phenotype approach) identified several genes by their putative functional gene variant

\section{REFERENCES}

1. Soltys J, Gong B, Kaminski HJ, Zhou Y, Kusner LL. Extraocular muscle susceptibility to myasthenia gravis: unique immunological burden, which associated with ophthalmoplegic cases (2). Prioritizing these genes by their expression levels in muscle showed they converged on muscle atrophy signaling and myosin II function pathways (2). These predictions were validated in gene expression studies using orbital muscle biopsies of MG cases compared to an independent control group without MG, pointing to dysregulated muscle networks in the ophthalmoplegic MG cases involving muscle atrophy and/or contractility as well as oxidative metabolism gene pathways (76). These pathways identified by gene variant burden, showed significant dysregulated correlations (which differed from controls) with known MG genes/pathways (70, 73), highlighting the importance of the MG context. The unmet need is developing a prognostic biomarker for the early detection of these cases.

\section{CONCLUDING REMARKS}

In juveniles with myasthenia, there are phenotypic differences amongst different populations in their ages at presentation, the proportions of ocular vs. generalized manifestation of MG, and in the treatment responsiveness of EOMs to immune therapies. Although ocular MG in younger children is often benign and self-limiting, indications are of genetically susceptible individuals who require a more aggressive approach with immune therapy to avoid chronic visual morbidity. There is a critical need for a prognostic biomarker to guide treatment approaches. In addition, clear knowledge gaps were identified; there is a lack of standardized use of descriptions of eye muscle involvement in juveniles with $\mathrm{MG}$, and poor descriptions of their responsiveness (or lack thereof) to immune therapies. The field will benefit from a collaborative response to these research gaps.

\section{AUTHOR CONTRIBUTIONS}

$\mathrm{JH}$ conceived the idea and wrote the first draft and prepared Table 2. AS performed the literature review and editorial input and prepared Table 1. TE and MN provided editorial input and prepared the figure and Table 3 . All authors contributed to the article and approved the submitted version.

\section{FUNDING}

$\mathrm{JH}$ and TE received funding from the South African (SA) National Research Funding Agency and MN is the recipient of a CReATe scholars award and a Carnegie Developing Emerging Academic Leaders (DEAL) award. This publication was made possible (in part) by a grant from Carnegie Corporation of New York and a L'Oréal-UNESCO For Women in Science South African Young Talents Award. AS is supported by an educational grant from Life Healthcare.

environment? Ann N Y Acad Sci. (2008) 1132:220-4. doi: 10.1196/annals.1 405.037

2. Nel M, Mulder N, Europa TA, Heckmann JM. Using whole genome sequencing in an african subphenotype of myasthenia 
gravis to generate a pathogenetic hypothesis. Front Genet. (2019) 10:136. doi: 10.3389/fgene.2019.00136

3. Munot P, Robb SA, Niks EH, Palace J. ENMC workshop study group. 2020. 242nd ENMC International Workshop: Diagnosis and management of juvenile myasthenia gravis Hoofddorp, the Netherlands, 1-3 March 2019. Neuromuscul Disord. (2020) 30:254-64. doi: 10.1016/j.nmd.2020. 02.001

4. Murai H, Yamashita N, Watanabe M, Nomura Y, Motomura M, Yoshikawa H. Characteristics of myasthenia gravis according to onset-age: Japanese nationwide survey. J Neurol Sci. (2011) 305:97-102. doi: 10.1016/j.jns.2011.03.004

5. Gui M, Luo X, Lin J, Li Y, Zhang M, Zhang X, et al. Long-term outcome of 424 childhood-onset myasthenia gravis patients. J Neurol. (2015) 262:82330. doi: 10.1007/s00415-015-7638-2

6. Feng HY, Wang HY, Liu WB, He XT, Huang X, Luo CM, et al. The high frequency and clinical feature of seronegative myasthenia gravis in Southern China. Neurol Sci. (2013) 34:919-24. doi: 10.1007/s10072-012-1159-x

7. Lee HN, Kang HC, Lee JS, Kim HD, Shin HY, Kim SM. 'Juvenile Myasthenia Gravis in Korea: Subgroup Analysis According to Sex and Onset Age. J Child Neurol. (2016) 31:1561-8. doi: 10.1177/0883073816666206

8. Wang W, Chen YP, Wang ZK, Wei DN, Yin L. A cohort study on myasthenia gravis patients in China. Neurol Sci. (2013) 34:175964. doi: 10.1007/s10072-013-1329-5

9. $\mathrm{Xu} \mathrm{L}$, Castro D, Reisch JS, Iannaccone ST. Response to treatment in pediatric ocular myasthenia gravis. Muscle Nerve. (2020) 61:22630. doi: 10.1002/mus.26745

10. Barraud C, Desguerre I, Barnerias C, Gitiaux C, Boulay C, Chabrol B. Clinical features and evolution of juvenile myasthenia gravis in a French cohort. Muscle Nerve. (2018) 57:603-09. doi: 10.1002/mus.25965

11. Heckmann JM, Hansen P, Van Toorn R, Lubbe E, Janse E, van Rensburg JMW, et al. The characteristics of juvenile myasthenia gravis among South Africans. S Afr Med J. (2012) 102:532-6. doi: 10.7196/SAMJ.5390

12. VanderPluym J, Vajsar FD, Jacob JK, Mah D, Grenier H. Clinical characteristics of pediatric myasthenia: a surveillance study. Pediatrics. (2013) 132:e939-44. doi: 10.1542/peds.2013-0814

13. Evoli A, Batocchi AP, Bartoccioni E, Lino MM, Minisci C, Tonali P. Juvenile myasthenia gravis with prepubertal onset. Neuromuscul Disord. (1998) 8:5617. doi: 10.1016/S0960-8966(98)00077-7

14. Popperud TH, Boldingh MI, Rasmussen M, Kerty E. Juvenile myasthenia gravis in Norway: Clinical characteristics, treatment, long-term outcome in a nationwide population-based cohort. Eur J Paediatr Neurol. (2017) 21:70714. doi: 10.1016/j.ejpn.2017.04.003

15. Jastrzebska A, Jastrzebski M, Ryniewicz B, Kostera-Pruszczyk A. Treatment outcome in juvenile-onset myasthenia gravis. Muscle Nerve. (2019) 59:54954. doi: 10.1002/mus.26445

16. Wong V, Hawkins BR, Yu YL. Myasthenia gravis in Hong Kong Chinese. Pediatric disease Acta Neurol Scand. (1992) 86:68-72. doi: 10.1111/j.1600-0404.1992.tb08056.x

17. Chou CC, Su IC, Chou IJ, Lin JJ, Lan SY, Wang YS, et al. Correlation of antiacetylcholine receptor antibody levels and long-term outcomes of juvenile myasthenia gravis in Taiwan: a case control study. BMC Neurol. (2019) 19:170. doi: 10.1186/s12883-019-1397-0

18. Ashraf VV, Taly AB, Veerendrakumar M, Rao S. Myasthenia gravis in children: a longitudinal study. Acta Neurol Scand. (2006) 114:11923. doi: 10.1111/j.1600-0404.2006.00646.x

19. Mansukhani SA, Bothun ED, Diehl NN, Mohney BG. Incidence and ocular features of pediatric myasthenias. Am J Ophthalmol. (2019) 200:24249. doi: 10.1016/j.ajo.2019.01.004

20. Vecchio D, Ramdas S, Munot P, Pitt M, Beeson D, Knight R, et al. Pediatric myasthenia gravis: Prognostic factors for drug free remission. Neuromuscul Disord. (2020) 30:120-27. 008. doi: 10.1016/j.nmd.2019.11.008

21. Somnier FE. Increasing incidence of late-onset anti-AChR antibody-seropositive myasthenia gravis. Neurology. (2005) 65:928-30. doi: 10.1212/01.wnl.0000176067.32186.a3

22. Lai $\mathrm{CH}$, Tseng HF. Nationwide population-based epidemiological study of myasthenia gravis in Taiwan. Neuroepidemiology. (2010) 35:66-71. doi: $10.1159 / 000311012$
23. Matsui N, Nakane S, Nakagawa $\mathrm{Y}$, Kondo K, Mitsui T, Matsumoto T. Increasing incidence of elderly onset patients with myasthenia gravis in a local area of Japan. J Neurol Neurosurg Psychiatry. (2009) 80:116871. doi: 10.1136/jnnp.2008.152637

24. Mombaur B, Lesosky MR, Liebenberg L, Vreede H, Heckmann JM. Incidence of acetylcholine receptor-antibody-positive myasthenia gravis in South Africa. Muscle. Nerve. (2015) 51:533-7. doi: 10.1002/mus.24348

25. Gattellari M, Goumas C, Worthington JM. A national epidemiological study of Myasthenia Gravis in Australia. Eur J Neurol. (2012) 19:141320. doi: 10.1111/j.1468-1331.2012.03698.x

26. Heckmann JM. Juvenile myasthenia gravis. Eur J Paediatr Neurol. (2017) 21:696. doi: 10.1016/j.ejpn.2017.07.011

27. Huang X, Liu WB, Men LN, Feng HY, Li Y, Luo CM, et al. Clinical features of myasthenia gravis in southern China: a retrospective review of 2,154 cases over 22 years. Neurol Sci. (2013) 34:911-7. doi: 10.1007/s10072-012-1157-Z

28. Huang X, Li Y, Feng H, Chen P, Liu W. Clinical Characteristics of Juvenile Myasthenia Gravis in Southern China. Front Neurol. (2018) 9:77. doi: 10.3389/fneur.2018.00077

29. Vanikieti K, Lowwongngam K, Padungkiatsagul T, Visudtibhan A, Poonyathalang A. (2018). Juvenile ocular myasthenia gravis: presentation and outcome of a large cohort. Pediatr Neurol. (2018) 87:36-41. doi: 10.1016/j.pediatrneurol.2018.06.007

30. Andrews PI, Massey JM, Howard JF, Sanders DB. Race, sex, puberty influence onset, severity, outcome in juvenile myasthenia gravis. Neurology. (1994) 44:1208-14. doi: 10.1212/WNL.44.7.1208

31. Yan C, Zhao R, Song J, Feng X, Xi J, Luo S, et al. Comparison of antiacetylcholine receptor profiles between Chinese cases of adult- and juvenileonset myasthenia gravis using cell-based assays. J Neuroimmunol. (2020) 349:577403. doi: 10.1016/j.jneuroim.2020.577403

32. Popperud TH, Boldingh MI, Brunborg C, Faiz KW, Heldal AT, Maniaol AH, et al. Juvenile myasthenia gravis in Norway: A nationwide epidemiological study. Eur J Paediatr Neurol. (2017) 21:312-7. doi: 10.1016/j.ejpn.2016. 09.001

33. Parr JR, Andrew MJ, Finnis M, Beeson D, Vincent A, Jayawant S. How common is childhood myasthenia? The UK incidence and prevalence of autoimmune and congenital myasthenia. Arch Dis Child. (2014) 99:53942. doi: 10.1136/archdischild-2013-304788

34. Chiang LM, Darras BT, Kang PB. Juvenile myasthenia gravis. Muscle Nerve. (2009) 39:423-31. doi: 10.1002/mus.21195

35. Niks EH, Kuks JB, Verschuuren JJ. Epidemiology of myasthenia gravis with anti-muscle specific kinase antibodies in The Netherlands. J Neurol Neurosurg Psychiatry. (2007) 78:417-8. doi: 10.1136/jnnp.2006. 102517

36. Finlayson S, Beeson D, Palace J. Congenital myasthenic syndromes: an update. Pract Neurol. (2013) 13:80-91. doi: 10.1136/practneurol-2012-0 00404

37. Cleary M, Williams GJ, Metcalfe RA. The pattern of extra-ocular muscle involvement in ocular myasthenia. Strabismus. (2008) 16:118. doi: 10.1080/15569520701830992

38. Europa TA, Nel M, Heckmann JM. Myasthenic ophthalmoparesis: Time To resolution after initiating immune therapies. Muscle Nerve. (2018) 58:54249. doi: $10.1002 /$ mus. 26172

39. Heckmann JM, Nel M, A. unique subphenotype of myasthenia gravis. Ann N Y Acad Sci. (2018) 1412:14-20. doi: 10.1111/nyas.13471

40. Kim JH, Hwang JM, Hwang YS, Kim KJ, Chae J. Childhood ocular myasthenia gravis. Ophthalmology. (2003) 110:145862. doi: 10.1016/S0161-6420(03)00460-3

41. Kraithat P, Hansapinyo L, Patikulsila P. Treatment Outcomes and Predictive Factors in Pediatric Ocular Myasthenia Gravis. J Med Assoc Thai. (2015) 98:883-8.

42. Ortiz S, Borchert M. Long-term outcomes of pediatric ocular myasthenia gravis. Ophthalmology. (2007) 115:1245-48. doi: 10.1016/j.ophtha.2007.10. 022

43. Jaretzki RJ, Barohn RM, Ernstoff HJ, Kaminski JC, Keesey AS, Penn DB. Myasthenia gravis: recommendations for clinical research standards. Task Force Med Scientific Advis Board Myasthenia Gravis Found Am Neurol. (2000) 55:16-23. doi: 10.1212/WNL.55.1.16 
44. Kupersmith MJ, Ying G. Ocular motor dysfunction and ptosis in ocular myasthenia gravis: effects of treatment. Br J Ophthalmol. (2005) 89:13304. doi: 10.1136/bjo.2004.063404

45. Heckmann JM, Rawoot A, Bateman K, Renison R, Badri M, A. single- blinded trial of methotrexate vs. azathioprine as steroid-sparing agents in generalized myasthenia gravis. BMC Neurol. (2011) 11:97. doi: 10.1186/1471-2377-11-97

46. Ferrara G, Mastrangelo G, Barone P, La Torre F, Martino S, Pappagallo G. Methotrexate in juvenile idiopathic arthritis: advice and recommendations from the MARAJIA expert consensus meeting. Pediatr Rheumatol Online J. (2018) 16:46. doi: 10.1186/s12969-018-0255-8

47. Finsterer J. Congenital myasthenic syndromes. Orphanet J Rare Dis. (2019) 14:57. doi: 10.1186/s13023-019-1025-5

48. Heckmann JM, Owen EP, Little F. Myasthenia gravis in South Africans: racial differences in clinical manifestations. Neuromuscul Disord. (2007) 17:92934. doi: 10.1016/j.nmd.2007.07.002

49. Mullaney P, Vajsar J, Smith R, Buncic JR. The natural history and ophthalmic involvement in childhood myasthenia gravis at the hospital for sick children. Ophthalmology. (2000) 107:504-10. doi: 10.1016/S0161-6420(99)00138-4

50. Huda S, Woodhall MR, Vincent A, Heckmann JM. Characteristics Of acetylcholine-receptor-antibody-negative myasthenia gravis in a South African cohort. Muscle Nerve. (2016) 54:1023-29. doi: 10.1002/mus.25154

51. Chan JW, Orrison WW. Ocular myasthenia: a rare presentation with MuSK antibody and bilateral extraocular muscle atrophy'. Br J Ophthalmol. (2007) 91:842-3. doi: 10.1136/bjo.2006.108498

52. Velonakis G, Papadopoulos VE, Karavasilis E, Filippiadis DK, Zouvelou V, MRI. evidence of extraocular muscle atrophy and fatty replacement in myasthenia gravis. Neuroradiology. (2021) 63:1531-8. doi: 10.1007/s00234-021-02753-4

53. Engel-Nitz NM, Boscoe A, Wolbeck R, Johnson J, Silvestri NJ. Burden of illness in patients with treatment refractory myasthenia gravis. Muscle Nerve. (2018) 58:99-105. doi: 10.1002/mus.26114

54. Rath J, Brunner I, Tomschik M, Zulehner G, Hilger E, Krenn M, et al. Frequency and clinical features of treatment-refractory myasthenia gravis. $J$ Neurol. (2020) 267:1004-11. doi: 10.1007/s00415-019-09667-5

55. Compston DA, Vincent A, Newsom-Davis J, Batchelor JR. Clinical, pathological, HLA antigen and immunological evidence for disease heterogeneity in myasthenia gravis. Brain. (1980) 103:579-601. doi: 10.1093/brain/103.3.579

56. Nel, M., and Heckmann, J. M. Epidemiology and genetics of myasthenia gravis. In, Myasthenia Gravis and Related Disorders edited Kaminski, H. and Kussner, L. Edition 3 (London: Humana Press). (2018).

57. Matsuki K, Juji T, Tokunaga K, Takamizawa M, Maeda H, Soda M, et al. antigens in Japanese patients with myasthenia gravis. J Clin Invest. (1990) 86:392-9. doi: 10.1172/JCI114724

58. Nomura Y, Ehara M, Matsuki K, Juji T, Segawa M, HLA. haplotype in myasthenia gravis (MG) of Japanese children. correlative studies between $\mathrm{mg}$ patients and their normal siblings and parents. Ann N Y Acad Sci. (1993) 681:581-3. doi: 10.1111/j.1749-6632.1993.tb22947.x

59. Chen WH, Chiu HC, Hseih RP. Association of HLA-Bw46DR9 combination with juvenile myasthenia gravis in Chinese. J Neurol Neurosurg Psychiatry. (1993) 56:382-5. doi: 10.1136/jnnp.56.4.382

60. Hawkins BR Yu YL, Wong V, Woo E, Ip MS, Dawkins RL. Possible evidence for a variant of myasthenia gravis based on HLA and acetylcholine receptor antibody in Chinese patients. Q J Med. (1989) 70:235-41. doi: 10.1093/oxfordjournals.qjmed.a068318

61. Popperud TH, Viken MK, Kerty E, Lie BA. Juvenile myasthenia gravis in Norway: HLA-DRB1*04:04 is positively associated with prepubertal onset. PLoS One. (2017) 12:e0186383. doi: 10.1371/journal.pone.0186383

62. Shinomiya, N., Nomura, Y., and Segawa, M. (2004). A variant of childhoodonset myasthenia gravis: HLA typing and clinical characteristics in Japan. Clin Immunol 0.110:154-8. doi: 10.1016/j.clim.2003.10.004

63. Zhu WH, Lu JH, Lin J, Xi JY, Lu J, Luo SS, et al. HLADQA $1 * 03: 02 / D Q B 1 * 03: 03: 02$ is strongly associated with susceptibility to childhood-onset ocular myasthenia gravis in Southern Han Chinese. J Neuroimmunol. (2012) 247:81-5. doi: 10.1016/j.jneuroim.2012. 03.018
64. Feng HY, Yang LX, Liu WB, Huang X, Qiu L, Li Y. The HLA-B*4601DRB1*0901 haplotype is positively correlated with juvenile ocular myasthenia gravis in a southern Chinese Han population. Neurol Sci. (2015) 36:113540. doi: 10.1007/s10072-015-2235-9

65. Nel M, Jalali Sefid Dashti M, Gamieldien J, Heckmann JM. Exome sequencing identifies targets in the treatment-resistant ophthalmoplegic subphenotype of myasthenia gravis. Neuromuscul Disord. (2017) 27:81625. doi: 10.1016/j.nmd.2017.06.009

66. Rautenbach RM, Pillay K, Murray ADN, Heckmann JM. Extraocular muscle findings in myasthenia gravis associated treatment-resistant ophthalmoplegia J Neuroophthalmol. (2017) 37:414-17. doi: 10.1097/WNO.0000000000000534

67. Europa TA, Nel M, Heckmann JM. A review of the histopathological findings in myasthenia gravis: Clues to the pathogenesis of treatmentresistance in extraocular muscles Neuromuscul Disord. (2019) 29:38187. doi: 10.1016/j.nmd.2019.03.009

68. Keene KR, van Vught L, van de Velde NM, Ciggaar IA, Notting IC, Genders SW, et al. The feasibility of quantitative MRI of extra-ocular muscles in myasthenia gravis and Graves' orbitopathy. NMR Biomed. (2020) 20:4407. doi: 10.1002/nbm.4407

69. Lueangaram S, Tritanon O, Siriyotha S, Vanikieti K, Padungkiatsagul T, Preechawat P. Radiological characteristics of extraocular muscles in myasthenia gravis patients with ocular manifestations: a case-control study. Clin Ophthalmol. (2021) 15:2279-85. doi: 10.2147/OPTH.S280508

70. Kaminski HJ, Himuro K, Alshaikh J, Gong B, Cheng G, Kusner LL. Differential RNA expression profile of skeletal muscle induced by experimental autoimmune myasthenia gravis in rats. Front Physiol. (2016) 7:524. doi: 10.3389/fphys.2016.00524

71. Andrade FH, McMullen CA. Lactate is a metabolic substrate that sustains extraocular muscle function. Pflugers Arch. (2006) 452:1028. doi: 10.1007/s00424-005-0010-0

72. Wai Man Y. Extraocular muscles have fundamentally distinct properties that make them selectively vulnerable to certain disorders. Neuromuscul Disord. (2005) 15:17-23. doi: 10.1016/j.nmd.2004.10.002

73. Hong Y, Liang X, Gilhus NE. AChR antibodies show a complex interaction with human skeletal muscle cells in a transcriptomic study. Sci Rep. (2020) 10:11230. doi: 10.1038/s41598-020-68185-x

74. Heckmann JM, Uwimpuhwe H, Ballo R, Kaur M, Bajic VB, Prince S, et al. functional SNP in the regulatory region of the decay-accelerating factor gene associates with extraocular muscle pareses in myasthenia gravis. Genes Immun. (2010) 11:1-10. doi: 10.1038/gene.2009.61

75. Nel M, Buys JM, Rautenbach R, Mowla S, Prince S, Heckmann JM. The African-387 C>T TGFB1 variant is functional and associates with the ophthalmoplegic complication in juvenile myasthenia gravis. J Hum Genet. (2016) 61:307-16. doi: 10.1038/jhg.2015.146

76. Europa TA, Nel M, Heckmann JM. Gene expression profiling of orbital muscles in treatment-resistant ophthalmoplegic myasthenia gravis. Orphanet J Rare Dis. (2020) 15:346. doi: 10.1186/s13023-020-01629-9

Conflict of Interest: The authors declare that the research was conducted in the absence of any commercial or financial relationships that could be construed as a potential conflict of interest.

Publisher's Note: All claims expressed in this article are solely those of the authors and do not necessarily represent those of their affiliated organizations, or those of the publisher, the editors and the reviewers. Any product that may be evaluated in this article, or claim that may be made by its manufacturer, is not guaranteed or endorsed by the publisher.

Copyright (c) 2022 Heckmann, Europa, Soni and Nel. This is an open-access article distributed under the terms of the Creative Commons Attribution License (CC BY). The use, distribution or reproduction in other forums is permitted, provided the original author(s) and the copyright owner(s) are credited and that the original publication in this journal is cited, in accordance with accepted academic practice. No use, distribution or reproduction is permitted which does not comply with these terms. 